\title{
ANÁLISIS DE LAS CREENCIAS EPISTEMOLÓGICAS DE ESTUDIANTES COLOMBIANOS SEGÚN VARIABLES SOCIODEMOGRÁFICAS Y EDUCATIVAS
}

\author{
Xavier G. Ordóñez ${ }^{1 *}$, Sonia J. Romero Martínez², Irma Rosete Fernández ${ }^{2}$ \\ 'Universidad Complutense de Madrid, ${ }^{2}$ Universidad a Distancia de Madrid
}

Recibido, septiembre 18/2014

Concepto evaluación, agosto 5/2015

Aceptado, septiembre 15/2015

\author{
Referencia: Ordóñez, X.G., Romero, S. J. \& Rosete Fernández, \\ I. (2016). Análisis de las creencias epistemológicas de estudiantes \\ colombianos según variables sociodemográficas y educativas. \\ Acta Colombiana de Psicología, 19(1), 57-67. DOI: 10.14718/ \\ ACP.2016.19.1.4
}

Resumen

\begin{abstract}
El objetivo principal del presente estudio es analizar y caracterizar la influencia de las variables educativas y sociodemográficas sobre el grado de desarrollo de las creencias epistemológicas (CE) en estudiantes universitarios y de último año de bachillerato. Para conseguir este objetivo se administró un instrumento llamado EQEBI, que mide las CE a una muestra de 1.387 alumnos en Bogotá (Colombia). La metodología utilizada fue cuantitativa y no experimental. Para comparar las CE de acuerdo con los grupos conformados según las variables analizadas, se utilizaron las pruebas estadísticas no paramétricas de Mann-Whitney y Kruskal-Wallis. Los resultados indican diferencias significativas en las CE por sexo, nivel socioeconómico y nivel educativo de los estudiantes y de sus padres. Sin embargo, no se encontraron diferencias según el entorno de procedencia (rural o urbano), ni por la repetición de curso. Este estudio tiene implicaciones para el diseño de programas educativos específicos, según las características de los alumnos, que favorezcan el desarrollo de las CE.

Palabras clave: creencias epistemológicas, EQEBI, educación, variables educativas y sociodemográficas.
\end{abstract}

\section{EPISTEMOLOGICAL BELIEFS OF COLOMBIAN STUDENTS ACCORDING TO EDUCATIONAL AND SOCIO-DEMOGRAPHIC VARIABLES}

\begin{abstract}
The main goal of the present study is to analyze and characterize the influence of educational and socio-demographical variables on the Epistemological Beliefs (EB) of senior year and university students. With this aim, an instrument that measures EB, called EQEBI, was applied to a sample of 1387 students in Bogota-Colombia. The methodology used was quantitative, non experimental, and the nonparametric statistics tests of Mann-Whitney and Kruskal-Wallis were used to compare the EB according to the groups formed by the variables analyzed. Results indicate significant differences in EB by sex, socioeconomic status and educational level of students and his parents, but no differences according to the environmental background (rural or urban) and grade repetition. This study has implications for the design of specific educational programs, according to the characteristics of students, to encourage the development of EB.

Key words: epistemological beliefs, EQEBI test, education, educational and socio-demographic variables.
\end{abstract}

* Xavier G. Ordóñez: Universidad Complutense de Madrid, Calle Rector Royo Villanova s/n, 28040 -Madrid, España Teléfono fijo: +34 913946147. xavor@ucm.es 


\title{
ANÁLISE DAS CRENÇAS EPISTEMOLÓGICAS DE ESTUDANTES COLOMBIANOS SEGUNDO VARIÁVEIS SOCIODEMOGRÁFICAS E EDUCATIVAS
}

\author{
Resumo
}

\begin{abstract}
O objetivo principal do presente estudo é analisar e caracterizar a influência de variáveis educativas e sociodemográficas sobre o grau de desenvolvimento das crenças epistemológicas (CE) em estudantes universitários e do último ano do ensino médio. Para atingir esse objetivo, administrou-se um instrumento chamado EQEBI, que mede as CE a uma amostra de 1.387 alunos em Bogotá (Colômbia). A metodologia utilizada foi quantitativa e não experimental. Para comparar as CE de acordo com os grupos formados segundo as variáveis analisadas, utilizaram-se as provas estatísticas não paramétricas de Mann-Whitney e Kruskal-Wallis. Os resultados indicam diferenças significativas nas CE por sexo, nível socioeconômico e nível educativo dos estudantes e de seus pais. Contudo, não se encontraram diferenças segundo o ambiente de procedência (rural ou urbano) nem pela repetição de curso. Este estudo tem implicações para o desenho de programas educativos específicos, conforme as características dos alunos, que favoreçam o desenvolvimento das $\mathrm{CE}$.

Palavras-chave: crenças epistemológicas, EQEBI, educação, variáveis educativas e sociodemográficas.
\end{abstract}

\section{INTRODUCCIÓN}

La epistemología estudia la naturaleza del conocimiento, la forma como este se obtiene y su alcance (Aulls y Lemay, 2013; Escalante, 2010). Una rama importante de la epistemología estudia las concepciones que tienen los estudiantes sobre el aprendizaje y el conocimiento. Algunas de las investigaciones sobre este tema se fundamentan en la teoría de que la fuente de las acciones y estrategias de los alumnos para lograr las metas de aprendizaje se basan en un conjunto de ideas o conceptos preconcebidos llamado Creencias Epistemológicas-CE (Angeli y Valanides, 2012; Martínez, Montero y Pedrosa, 2010; Schommer, Beuchat y Hernández, 2012; Terzi, Çetin y Eser, 2012). Es por ello que el análisis de las CE y de los factores que pueden influir en su desarrollo resulta fundamental, tanto para educadores como para psicólogos interesados en mejorar las acciones y estrategias de aprendizaje de los alumnos en todos los niveles educativos.

Comúnmente, las $\mathrm{CE}$ han sido analizadas desde una tradición fenomenográfica (Marton, 1981) centrada en el análisis de protocolos verbales con respuestas breves y entrevistas semiestructuradas, con su posterior categorización. Sin embargo, también se han utilizado otros métodos para investigar las CE, por ejemplo, cuestionarios y tareas cuasi-experimentales, siendo estas últimas la perspectiva metodológica que se ha utilizado en el presente trabajo. Las primeras investigaciones desde este punto de vista se atribuyen a Perry en los años 70 (Acosta, 2009; Escalante, 2010; Rodríguez, 2005), quien observó que el desarrollo epistemológico evoluciona desde visiones más absolutistas y dualistas del conocimiento hacia otras más relativas basadas en la observación y el razonamiento (Brownlee, 2003; Herrón, 2010; King y Magun, 2009). Las ideas de
Perry ejercieron un gran impacto en otros investigadores, quienes empezaron a considerar el carácter evolutivo de las CE.

Siguiendo esta corriente evolutiva, Schommer (1990) propone que las CE evolucionan en distintos grados de sofisticación y presenta un sistema de cinco dimensiones que las componen. Tres de ellas se refieren a las creencias sobre el conocimiento, y las dos restantes, a concepciones sobre el aprendizaje (Alsamie e Ismail, 2005; Hofer y Pintrich, 1997; García y Sebastián, 2011; Gómez y Silas, 2012; Hernández y Maquilón, 2011):

1. Certeza del Conocimiento (CC), que varía desde el polo del conocimiento como algo cierto, absoluto e inmutable hasta su consideración como algo tentativo y sujeto a cambios.

2. Simplicidad del Conocimiento (SC), que oscila entre la concepción del conocimiento como aislado y simple frente a la creencia de que es complejo e integrado.

3. Autoridad Omnisciente (AO), que varía desde la creencia de que la fuente del conocimiento está en los expertos hasta la creencia de que está en la razón.

4. Velocidad de Aprendizaje (VA), que oscila entre la concepción del proceso de aprendizaje como algo rápido vs. lento.

5. Habilidad Innata (HI), que varía entre la creencia de que el aprendizaje está genéticamente determinado y la de que es un proceso de construcción ambiental.

Según Schommer (1990), las dimensiones que componen las $\mathrm{CE}$ son independientes, de tal forma que una misma persona puede tener unas creencias sofisticadas en una dimensión y sencillas en otra (Terrazas y Frenay, 2009; Vizcaíno, Otero y Mendoza, 2013). Aunque esta es la clasificación más utilizada de las CE, tanto en las investigaciones como en el desarrollo de instrumentos de 
medición, también se han descrito otros sistemas de clasificación (Pozo y Scheuer, 1999; Säljö, 1979).

Dentro del interés de los investigadores por conocer y promocionar un mejor desarrollo de las $\mathrm{CE}$, destaca el estudio de aspectos o factores que pueden influir en las mismas. La mayor parte de estos estudios incluye el análisis de dos tipos de variables: variables socio-demográficas como la edad, el sexo, el lugar de procedencia y el nivel socio-económico, y variables educativas como el máximo nivel de estudios alcanzado por los alumnos y sus padres, el tipo de colegio o las estrategias de aprendizaje, entre otras. A continuación se presentarán los resultados de algunos estudios previos en los que se utilizaron variables similares a las seleccionadas en el presente trabajo:

1. Variables socio-demográficas. Varios estudios han evidenciado que las características personales como el sexo o la edad influyen en el desarrollo de las CE. En el caso del sexo, hay un patrón de resultados mixto (Özkan y Tekkaya, 2011; Walter, 2008) pues hay investigadores que no encontraron diferencias significativas (Buehl, Alexander y Murphy, 2002; Chan y Elliott, 2002), pero otros señalaron diferencias en varias dimensiones de las CE. Por ejemplo, se ha documentado que las mujeres muestran unas creencias más sofisticadas en cuanto a la velocidad del aprendizaje, pero menos desarrolladas respecto a la simplicidad del conocimiento (Cano, 2005; Hofer, 2000; Paulsen y Wells, 1998; Schommer, Calvert, Gariglietti y Bajaj, 1997; Schommer y Dunnel, 1994; Walker, 2007). Otros autores han encontrado que los hombres tienen creencias menos sofisticadas que las mujeres en certeza del conocimiento y autoridad omnisciente (Bendixen, Schraw y Dunkle, 1998; Hofer, 2000).

Respecto a la edad, existe consenso en los resultados: los autores reportan un cambio evolutivo que va desde creencias menos sofisticadas a otras más desarrolladas a medida que se incrementa la edad (Conley, Pintrich, Vekiri y Harrison, 2004; García y Sebastián, 2011; Paechter et al., 2013). Otros autores han encontrado que las CE dependen de la adquisición de experiencias en contextos de aprendizaje (Lahtinen y Pehkonen, 2013; Taha y El-Habbal, 2013), y aún más, se ha encontrado que las CE se desarrollan en un proceso recursivo que dura toda la vida (Schommer, 1990).

Por otra parte, se ha encontrado que la influencia de otras variables como el nivel socio-económico o la zona de procedencia se han estudiado en menor medida que las características personales. Respecto al estatus socioeconómico, Cano y Cardelle (2008) han encontrado que las personas de estrato socio-económico bajo tienen unas creencias más ingenuas que el promedio, tendiendo a pensar que el conocimiento reside en figuras de autoridad externas.
De forma similar, Ozkal, Tekkaya, Sungur, Cakiroglu y Cakiroglu (2011) observaron que los alumnos provenientes de familias con un nivel socioeconómico alto tendían a ver el conocimiento como algo tentativo y auto-orientado.

2. Variables educativas. Uno de los pioneros en el estudio de la relación de variables educativas y el desarrollo de las CE ha sido Säljö (1979) quien estableció y comprobó la hipótesis de la existencia de una relación significativa entre las CE y las estrategias de aprendizaje. También hay consenso en los resultados de las investigaciones respecto a que un mayor nivel educativo tanto de los propios estudiantes como de sus padres incrementa el desarrollo de las CE (Baldwin y Alsumait, 2013; Walker, 2007). Por ejemplo, Mason, Boldrin y Zurlo (2006) señalaron un cambio decisivo en el desarrollo de las CE entre los estudiantes de primaria ( $5^{\circ}$ grado) y secundaria ( $8^{\circ}$ grado). También Önen (2011) indicó diferencias significativas entre los estudiantes a medida que el grado escolar incrementaba. Marzooghi, Fouladchang y Shemshiri (2007) concluyeron que los estudiantes del primer año de universidad tenían creencias más simples y pensaban que el conocimiento se adquiría más rápido respecto a los del cuarto año. De forma similar, Jehng, Johnson y Anderson (1993) hallaron que los alumnos de pregrado tenían unas CE menos desarrolladas al compararlas con las de alumnos de posgrado.

Respecto a la influencia del nivel educativo de los padres, Schommer (1990) encontró que cuanto mayor era el nivel educativo de ambos padres, mayor era la probabilidad de que los hijos desarrollaran un sistema epistemológico más sofisticado. Sin embargo, esta importante variable ha sido muy poco analizada en la literatura científica. En otra investigación, Topçu y Yilmaz (2009) hallaron diferencias en la VA según el nivel educativo de la madre; en concreto, estudiantes con madres de mayor nivel educativo tendían a percibir el aprendizaje como un proceso lento y gradual.

Por otra parte, algunos estudios se han centrado en evaluar si existen diferencias en las CE de acuerdo con el área de estudio de las personas, encontrando que, efectivamente, las creencias varían según el tipo de dominio (Llinares, García, Martí y Casino, 2013). Así, los alumnos de ciencias sociales, humanidades o artes presentan unas creencias más sofisticadas en CC, SC y AO, en oposición a los alumnos de matemáticas, ingeniería y negocios (Hofer, 2000; Jehng, Johnson y Anderson, 1993; Schommer y Orpha, 2013).

Con el ánimo de profundizar en los resultados de los estudios antes presentados, el objetivo general de este trabajo es analizar la influencia de variables sociodemográficas y educativas sobre el nivel de desarrollo de las creencias epistemológicas en una amplia muestra de estudiantes colombianos. Para conseguir el objetivo se han seleccionado, 
medido y analizado las siguientes variables: sexo (varones, mujeres), tipo de institución (privada, pública), repetición de curso durante la secundaria (si, no), entorno del que provienen (rural, urbano), nivel de estudios (secundaria, universitario), nivel educativo de los padres (primaria, secundaria, universitario, postgrado), estrato social (1 a 6, donde 1 es muy bajo y 6 es muy alto) y área de estudio (Psicología, Filosofía, Filología, Ingeniería, Economía y Bachillerato). Un objetivo secundario ha sido el estudio descriptivo de las variables analizadas para lograr una caracterización de los alumnos. Siguiendo los resultados de las investigaciones previas, las hipótesis que orientan esta investigación son:

1. En cuanto a la influencia de las variables sociodemográficas: a) habrá diferencias significativas en la EB de acuerdo con las características personales como el sexo y la edad (Bendixen, Schraw y Dunkle; 1998; Cano, 2005; Hofer, 2000; Paulsen y Wells, 1998; Schommer y Dunnel, 1994), b) no habrá diferencias según el entorno de procedencia del estudiante (Topçu y Yilmaz, 2009), y c) las CE estarán más desarrolladas en los estudiantes de mayor nivel socio-económico (Conley et al., 2004 y Ozkal et al., 2011).

2. En cuanto a la influencia de las variables educativas: a) habrá diferencias significativas entre los estudiantes de escuelas públicas y privadas (Ismail, Hassan, Muhamad, Ali y Konting, 2013), b) los estudiantes que repiten algún curso durante la escuela secundaria tendrán unas creencias menos desarrolladas (Hofer, 2000; Schommer et al., 1997; Paulsen y Wells, 1998), c) las creencias estarán más desarrolladas en los estudiantes con padres de mayor nivel educativo (Schommer, 1990; Topçu y Yilmaz, 2009), d) las CE de los estudiantes de secundaria estarán menos desarrolladas que las de los estudiantes universitarios (Baldwin y Alsumait, 2013; Walker, 2007), y d) no habrá diferencias significativas en las CE según el área de estudio (Hofer, 2000; Schommer y Orpha, 2013).

\section{MÉTODO}

\section{Participantes}

Un total de 1.387 alumnos participaron en el estudio, 890 estudiantes universitarios y 497 en el último año de bachillerato. El 54,7\% de los alumnos eran hombres y el $45,3 \%$, mujeres. En cuanto a la edad, la media era de 18.2 años $(D T=2.6)$, las edades mínima y máxima eran de 14 y 40 años, respectivamente. Se utilizó un muestreo por conveniencia, motivo por el cual la muestra no es probabi- lística. Los estudiantes participaron de forma voluntaria y mediante consentimiento informado. Se aseguró el uso de los datos para fines de investigación y la confidencialidad de la información.

\section{Instrumentos}

Para la realización de este estudio se aplicó un instrumento de auto-informe, compuesto por un bloque introductorio en el que se recoge información sobre las variables sociodemográficas y educativas seguido de un segundo bloque que contiene el EQEBI, un instrumento en español, tipo Likert, para medir las CE, propuesto por Ordoñez, Ponsoda, Abad y Romero (2009). El EQEBI consta de las siguientes cuatro dimensiones: Certeza del Conocimiento (cuatro ítems), Simplicidad del Conocimiento (cuatro ítems), Velocidad del Aprendizaje (once ítems) y Habilidad Innata (ocho ítems), con unas fiabilidades ( $\alpha$ de Cronbach) de .88, .70, .67 y .81 respectivamente (Ordoñez et al., 2009). En cuanto a la validez de las puntuaciones, los autores realizan dos estudios independientes de análisis factorial confirmando la estructura de cuatro dimensiones independientes en ambos casos. Adicionalmente, Ordoñez et al. (2009) calibran los ítems con el modelo de respuesta graduada, mostrando que los ítems que componen la prueba cuentan con adecuadas propiedades psicométricas.

\section{Procedimiento}

Como primer paso de la presente investigación se realizó el análisis de la documentación teórica que sustenta este proyecto, y con base en dicho análisis se realizó la selección de variables a emplear y se diseñó el instrumento utilizado para recoger la información. En segundo lugar, se realizó el contacto inicial con los colegios y universidades que, por su relación con algunos miembros del equipo de investigación, podrían colaborar para la aplicación del instrumento. Tras la confirmación de dichas instituciones el instrumento fue facilitado a los estudiantes en formato de lápiz y papel durante el tiempo de clase a través de los profesores de los centros educativos que participaron en el estudio. Uno de los miembros del equipo de investigación estaba presente en el momento de aplicación. Una vez recogidos los instrumentos se procedió al procesamiento previo y análisis de los datos, el cual incluyó varias fases: a) preparación de códigos, b) estructuración del archivo, c) grabación de datos, d) revisión y corrección de datos erróneos, e) prueba de supuestos y d) análisis de datos, que a su vez incluyó, tanto la descripción de variables como la comparación de grupos de acuerdo con las variables previamente seleccionadas. 


\section{Análisis de datos}

Esta investigación puede definirse como cuantitativa, no experimental. Se utilizó una metodología descriptiva y comparativa (Ato, López y Benavente, 2013). Las variables analizadas (sociodemográficas, educativas y las creencias epistemológicas) fueron evaluadas mediante un instrumento de auto-informe cuyas características se especificarán en el siguiente epígrafe. Los análisis de datos se realizaron en SPSS 20. Debido al incumplimiento de los supuestos requeridos para la correcta utilización de la estadística paramétrica, la comparación entre grupos se llevó a cabo mediante las pruebas no paramétricas U de Mann-Whitney (dos grupos) y H de Kruskal-Wallis (más de dos grupos). El tamaño del efecto se calculó mediante el estadístico $r$, apropiado para este tipo de análisis (Fritz, Morris y Ritchler, 2012).

\section{RESULTADOS}

A continuación se presentan los resultados del estudio: en primer lugar, se presenta una descripción de las características socio-demográficas y educativas de la muestra. En segundo lugar, se presentan los resultados de la comprobación de supuestos para la utilización de la estadística paramétrica. En tercer lugar, se presentan los resultados del análisis de las diferencias en las CE de los alumnos de acuerdo al sexo, tipo de colegio, repetición de curso, sector de procedencia, nivel de estudios, escolaridad de los padres, estrato socioeconómico y área de estudio.

Características de la muestra

Respecto a las características de la muestra, un $62 \%$ de los alumnos proviene de colegios de carácter privado y un $37.4 \%$ de colegios públicos. Un $20.2 \%$ de la muestra repitió algún curso en el colegio y el $91.3 \%$ proviene de entornos urbanos. La distribución según el estrato social es la siguiente: estrato 1 (4.6\%), 2(13.6\%), 3 (36.3\%), 4 (29.4\%), $5(11.3 \%)$ y $6(4.8 \%)$. Respecto al nivel educativo de los padres, la distribución es la siguiente: primaria (13.9\%), secundaria $(32.5 \%)$, universitaria $(32.7 \%)$ y postgrado (20.9\%). Con respecto al nivel educativo de las madres, la distribución porcentual es: primaria $(13.4 \%)$, secundaria (40.3\%), universitaria (31.9\%) y postgrado (14.4\%).

Comprobación de supuestos paramétricos

Respecto a la comprobación de supuestos para la correcta utilización de la estadística paramétrica se puede observar en las Tablas 1 y 2 que no se cumple el supuesto de normalidad en ninguno de los grupos a analizar (excepto en Filosofía y en algunas escalas de Bachillerato). Debido al incumplimiento de este supuesto se decidió utilizar la estadística no paramétrica para la comparación de grupos.

Tabla 1.

Prueba de Shapiro-Wilk para los grupos conformados por sexo, tipo de institución, repetición de curso, escolaridad del padre y de la madre

\begin{tabular}{|c|c|c|c|c|c|c|c|c|c|c|c|c|c|c|}
\hline \multirow[b]{2}{*}{$\mathrm{CE}$} & \multicolumn{2}{|l|}{ Sexo } & \multicolumn{2}{|l|}{ Tipo } & \multicolumn{2}{|c|}{ Repetición } & \multicolumn{4}{|c|}{ Escolaridad Padrea } & \multicolumn{4}{|c|}{ Escolaridad madrea } \\
\hline & $\mathrm{V}$ & M & Pub. & Priv. & $\mathrm{Si}$ & No & 1 & 2 & 3 & 4 & 1 & 2 & 3 & 4 \\
\hline Total & $.876^{*}$ & $.847^{*}$ & $.861 *$ & $.864 *$ & $.901 *$ & $.851^{*}$ & $.910^{*}$ & $.870^{*}$ & $.851^{*}$ & $.839 *$ & $.921^{*}$ & $.861 *$ & $.859 *$ & $.811^{*}$ \\
\hline VA & $.887^{*}$ & $.855^{*}$ & $.875^{*}$ & $.900 *$ & $.935^{*}$ & $.855^{*}$ & $.926^{*}$ & $.890^{*}$ & $.927^{*}$ & $.855^{*}$ & $.910^{*}$ & $.890^{*}$ & $.876^{*}$ & $.813^{*}$ \\
\hline HI & $.963^{*}$ & $.940^{*}$ & $.959 *$ & $.938^{*}$ & $.960^{*}$ & $.949 *$ & $.964 *$ & $.959 *$ & $.958^{*}$ & $.958^{*}$ & $.959 *$ & $.960 *$ & $.947^{*}$ & $.947^{*}$ \\
\hline $\mathrm{CC}$ & $.946^{*}$ & $.929 *$ & $.914 *$ & $.937^{*}$ & $.942 *$ & $.962 *$ & $.958^{*}$ & $.945^{*}$ & $.968^{*}$ & $.954 *$ & $.952 *$ & $.947^{*}$ & $.965^{*}$ & $.915^{*}$ \\
\hline $\mathrm{SC}$ & $.979 *$ & $.985^{*}$ & $.984 *$ & $.979 *$ & $.982 *$ & $.983 *$ & $.960 *$ & $.978^{*}$ & $.977 *$ & $.978^{*}$ & $.962 *$ & $.977 *$ & $.976^{*}$ & $.973 *$ \\
\hline
\end{tabular}

Nota: $* \mathrm{p}<.01$

a: 1. Primaria 2. Secundaria 3. Universidad 4. Postgrado 
Tabla 2.

Prueba de Shapiro-Wilk para los grupos conformados por procedencia, estrato social y área de estudio

\begin{tabular}{|c|c|c|c|c|c|c|c|c|c|c|c|c|c|c|}
\hline & \multicolumn{2}{|c|}{ Procedencia } & \multicolumn{6}{|c|}{ Estrato social } & \multicolumn{6}{|c|}{ Área de estudioa } \\
\hline & Rural & Urb. & 1 & 2 & 3 & 4 & 5 & 6 & 1 & 2 & 3 & 4 & 5 & 6 \\
\hline Total & $.866^{*}$ & $.875^{*}$ & $.939 *$ & $.905^{*}$ & $.853^{*}$ & $.858^{*}$ & $.914 *$ & $.681^{*}$ & $.847^{*}$ & $.853^{*}$ & .939 & $.975^{*}$ & $.805^{*}$ & $.887^{*}$ \\
\hline VA & $.939 *$ & $.847^{*}$ & $.873^{*}$ & $.920^{*}$ & $.890^{*}$ & $.876^{*}$ & $.859 *$ & $.734^{*}$ & $.853 *$ & $.842 *$ & .956 & $.762 *$ & $.839^{*}$ & $.892 *$ \\
\hline HI & $.948^{*}$ & $.937 *$ & $.966^{*}$ & $.970 *$ & $.969 *$ & $.967^{*}$ & $.972 *$ & $.909^{*}$ & $.942 *$ & $.948^{*}$ & .921 & .915 & $.854 *$ & $.955^{*}$ \\
\hline $\mathrm{CC}$ & $.972 *$ & $.878^{*}$ & $.933 *$ & $.935^{*}$ & $.939 *$ & $.955^{*}$ & $.977^{*}$ & $.922 *$ & $.933^{*}$ & $.952 *$ & .970 & $.781 *$ & .929 & $.931^{*}$ \\
\hline $\mathrm{SC}$ & $.982 *$ & $.973 * *$ & $.937^{*}$ & $.982 *$ & $.983 *$ & $.969 *$ & $.964 *$ & $.899 *$ & $.968^{*}$ & $.966^{*}$ & .898 & .943 & .938 & $.983 *$ \\
\hline
\end{tabular}

Nota: $* \mathrm{p}<.01$

a: 1. Ingeniería 2. Psicología 3. Filosofía 4. Filología 5. Economía 6. Bachillerato

\section{Diferencias en las CE según el sexo}

Hipótesis estadísticas: la hipótesis nula es que la tendencia central de las puntuaciones de las CE de hombres y mujeres es la misma $\left[\mathrm{H}_{0}: \mathrm{E}(\mathrm{H})=\mathrm{E}(\mathrm{M})\right]$; la hipótesis alterna es que la tendencia central es diferente $\left[\mathrm{H}_{1}: \mathrm{E}(\mathrm{H}) \neq \mathrm{E}(\mathrm{M})\right]$.

El contraste U de Mann-Whitney indica que se puede rechazar la hipótesis nula en el caso de la escala CC ( $U$ $=194810, Z=-2.927, p=.003)$. Observando la mediana se aprecian unas creencias más elaboradas en los varones $(\mathrm{Mdn}=9)$ que en las mujeres $(\mathrm{Mdn}=10)$ Sin embrago, el tamaño del efecto fue bajo $(r=.081)$. En el resto de las escalas y en la puntuación total no se pudo rechazar la hipótesis nula: total $(U=169002.5, Z=-1.108, p=.268)$, $\mathrm{SC}(U=209854, Z=-.660, p=.509), \mathrm{HI}(U=203869, Z=$ $-.133, p=.894)$ y VA $(U=191208.5, Z=-1.506, p=.132)$. Diferencias en las CE según el tipo de colegio

Hipótesis estadísticas: la hipótesis nula es que la tendencia central de las puntuaciones en las CE de alumnos procedentes de colegios privados es igual a la de los alumnos de colegios públicos $\left[\mathrm{H}_{0}: \mathrm{E}(\mathrm{Pr})=\mathrm{E}(\mathrm{Pu})\right]$; la hipótesis alterna es que la tendencia central es diferente $\left[\mathrm{H}_{1}: \mathrm{E}(\mathrm{Pr}) \neq \mathrm{E}(\mathrm{Pu})\right]$.

El contraste, $\mathrm{U}$ de Mann-Whitney mostró una diferencia estadísticamente significativa entre estudiantes que proceden de colegios públicos y privados tanto en las puntuaciones totales del test $(U=214322, Z=-3.093, p=.002)$ como en las escalas VA $(U=140693, Z=-2.798, p=.005), \mathrm{SC}(U$ $=144726, Z=-4.194, p=.003$ y CC $(U=125649.5, Z=$ $7.096, p<.000)$. Dichas diferencias indican unas creencias totales más sofisticadas en alumnos que han estudiado en colegios privados $(\mathrm{Mdn}=57)$ que en los públicos $(\mathrm{Mdn}=$
58). Respecto a la escala VA nuevamente hay unas creencias más desarrolladas en alumnos de colegios privados $(\mathrm{Mdn}=21)$ respecto a los de instituciones públicas (Mdn $=22$ ). Lo mismo ocurre con las escalas SC (privados $=9$ vs públicos $=10)$ y CC (privados $=10$ vs públicos $=11)$ El tamaño del efecto fue bajo-moderado $(r=.077, r=.082$, $r=.121$ y $r=.206$, respectivamente). En la escala HI no se hallaron diferencias significativas $(U=151750.5, Z=$ $-1.630, p=.103)$.

Diferencias en las CE según repetición de curso

Hipótesis estadísticas: la hipótesis nula es que la tendencia central de las puntuaciones en las CE de alumnos que han repetido curso y los que no han repetido es la misma $\left[\mathrm{H}_{0}\right.$ : $\mathrm{E}(\mathrm{rep})=\mathrm{E}($ no rep) $]$; la hipótesis alterna es que la tendencia central de las puntuaciones es diferente $\left[\mathrm{H}_{1}\right.$ : $\mathrm{E}($ rep $) \neq \mathrm{E}$ (no rep)].

Según el contraste U de Mann-Whitney no fue posible rechazar la hipótesis nula, ni para el total del test $(U=87855$, $Z=-1.078, p=.281)$, ni para ninguna las subescalas: VA $(U=105402.5, Z=-.815, p=.415), \mathrm{SC}(U=113411.5, Z$ $=-.495, p=.621), \mathrm{HI}(U=106294.5, Z=-.114, p=.910)$ у CC $(U=106647, Z=-1.424, p=.154)$.

Diferencias en las CE según el entorno de procedencia

Hipótesis estadísticas: la hipótesis nula es que la tendencia central de las puntuaciones en las CE de estudiantes que provienen de entornos rurales y urbanos es la misma $\left[\mathrm{H}_{0}: \mathrm{E}(\mathrm{R})=\mathrm{E}(\mathrm{U})\right]$; la hipótesis alterna es que la tendencia central de las puntuaciones es diferente $\left[\mathrm{H}_{1}: \mathrm{E}(\mathrm{R}) \neq \mathrm{E}(\mathrm{U})\right]$.

Según el contraste $U$ de Mann-Whitney no fue posible rechazar la hipótesis nula, ni para el total del test $(U=$ 
50673.5, $Z=-.520, p=.603)$, ni para ninguna de las subescalas: VA $(U=56913, Z=-1.477, p=.140), \mathrm{SC}(U=$ 66268.5, $Z=-.106, p=.916), \mathrm{HI}(U=59957, Z=-.816$, $\mathrm{p}=.414)$ y CC $(U=61696.5, Z=-1.419, p=.156)$.

\section{Diferencias en las CE según el nivel de estudios}

Hipótesis estadísticas: la hipótesis nula es que la tendencia central de las puntuaciones en las CE de estudiantes de universidad y de último año de bachillerato es la misma $\left[\mathrm{H}_{0}: \mathrm{E}(\mathrm{B})=\mathrm{E}(\mathrm{U})\right]$; la hipótesis alterna es que la tendencia central es diferente $\left[\mathrm{H}_{1}: \mathrm{E}(\mathrm{B}) \neq \mathrm{E}(\mathrm{U})\right]$.

Según la prueba U de Mann-Whitney existe evidencia para rechazar la hipótesis nula, tanto en las puntuaciones totales del test $(U=137217, Z=-3.485, p<.000)$, como en las escalas VA $(U=169372, Z=-2.945, p=.003)$, SC $(U=181985, Z=-3.302, p=.001)$ y CC $(U=129491.5$, $Z=-10.973, p<.000)$. Las diferencias en la tendencia central indican que los alumnos universitarios tienen unas creencias más elaboradas que los alumnos de último año de bachillerato con medianas de 56 y 58 , respectivamente, en la puntuación total; medianas de 21 y 22 , respectivamente, en la escala VA, y medianas de 9 y 10 , respectivamente, en las escalas CC y SC. El tamaño del efecto fue bajo-moderado $(r=.100 ; r=.083, r=.091$ y $r=.301)$. En la escala HI no se pudo rechazar la hipótesis nula $(U=191422, Z=$ $-.343, p=.732)$.

Diferencias en las CE según el nivel de estudios del padre y la madre

Hipótesis estadísticas: la hipótesis nula es que la tendencia central de las puntuaciones en las CE es la misma en estudiantes con padres de diversos niveles educativos $\left[\mathrm{H}_{0}: \mathrm{E}(\right.$ primaria $)=\mathrm{E}($ secundaria $)=\mathrm{E}($ universidad $)=$ $\mathrm{E}$ (postgrado)]; la hipótesis alterna es que la tendencia central es diferente $\left[\mathrm{H}_{1}\right.$ : $\mathrm{E}($ primaria $) \neq \mathrm{E}($ secundaria $) \neq$ $\mathrm{E}$ (universidad) $\neq \mathrm{E}$ (post-grado) $]$.

El contraste $\mathrm{H}$ (Kruskal-Wallis) mostró diferencias estadísticamente significativas de acuerdo con la escolaridad del padre, tanto en las puntuaciones totales del test $(H=$ $13.49, g l=3, p=.004)$, como en las escalas $\mathrm{SC}(H=24.68$, $g l=3, p<.000)$ y CC $(H=92.57, g l=3, p<.000)$. En las demás escalas no hay diferencias significativas: $\mathrm{HI}(H=$ $3.752, g l=3, p=.290)$ y VA $(H=3.642, g l=3, p=.303)$.

Para saber entre qué grupos concretos existen diferencias se realizó un análisis post-hoc usando el test de Mann-
Whitney con corrección de Bonferroni. Esta prueba indica que las diferencias de la puntuación total ocurren entre las categorías primaria-universidad $(U=26656, Z=-3.273$, $p=.001, r=.290)$ y primaria-posgrado $(U=173125, Z=$ $-2.954, p=.003, r=.338$ ).

En la escala SC hay diferencias entre las categorías primaria-bachillerato $(U=33536.5, Z=-2.774, p=.006$, $r=.112)$, primaria-universidad $(U=29274.5, Z=-4.505$, $p<.000, r=.194)$ y primaria-posgrado $(U=18823, Z=$ $-4.084, p<.000, r=.193)$. Finalmente, en la escala CC hay diferencias entre las categorías bachillerato-universidad $(U=65009, Z=-6.593, p<.000, r=.290)$, bachilleratoposgrado $(U=38730, Z=-6,370, p<.000, r=.338)$, primaria-universidad $(U=23716.5, Z=-7.078, p<.000$, $r=.227)$ y primaria-posgrado $(U=13954 ; Z=-7.062, p$ $<.000, r=.244)$. Las diferencias muestran creencias más elaboradas en alumnos con padres que tienen mayor nivel educativo, como se puede observar en la Tabla 3.

El contraste $\mathrm{H}$ también mostró una diferencia significativa de acuerdo con la escolaridad de la madre, tanto en las puntuaciones totales del test $(H=11.937, g l=3, p=.008)$, como en las escalas SC $(H=18.367, g l=3, p<.000)$ y $\mathrm{CC}(H=78.089, g l=3, p<.000)$. En las demás escalas no hay diferencias: $\mathrm{HI}(H=2.438, g l=3, p=.487)$ y VA $(H=2.012, g l=3, p=.570)$.

El análisis post-hoc indica que dichas diferencias ocurren en la puntuación total del test entre las categorías primaria-universidad $(U=26456.5, Z=-2.870, p=.004, r$ $=.123)$ y primaria-posgrado $(U=11826.5, Z=-2.727, p=$ $.006, r=.148)$. En la escala SC ocurren entre bachilleratouniversidad $(U=94630, Z=-3.355, p=.001, r=.109)$, bachillerato-posgrado $(U=42658, Z=-3.032, p=.002$, $r=.113)$, primaria-universidad $(U=32032, Z=-2.623, p$ $=.009, r=.108)$ y primaria-posgrado $(U=14438.5, Z=$ $-2.595, p=.009, r=.135)$. En la escala CC ocurren entre primaria-universidad $(U=23990.5, Z=-6.054, p<.000$, $\mathrm{r}=.254)$, primaria-posgrado $(U=10395.5, Z=-5.324, \mathrm{p}<$ $.000, r=.284)$, bachillerato-universidad $(U=72938.0, Z$ $=-6.538, p<.000, r=.219) \mathrm{y}$ bachillerato-posgrado $(U$ $=31731, Z=-5.216, p<.000, r=.200)$. Las diferencias indican creencias más elaboradas en alumnos con madres que cuentan con un mayor nivel educativo, como se puede observar en la Tabla 3. 
Tabla 3.

Puntuación mediana según escolaridad del padre y de la madre para el total, SC y CC

\begin{tabular}{lccccccccccccc}
\hline EQEBI & \multicolumn{3}{c}{ Total } & \multicolumn{3}{c}{ SC } \\
\hline Nivel & 1 & 2 & 3 & 4 & 1 & 2 & 3 & 4 & 1 & 2 & 3 & 4 \\
Padre & 59 & 58 & 56 & 56 & 10 & 9 & 9 & 9 & 12 & 11 & 9 & 9 \\
Madre & 59 & 58 & 56 & 56 & 9 & 9 & 9 & 8 & 11 & 11 & 9 & 9 \\
\hline
\end{tabular}

Diferencias en las CE según el estrato social

Hipótesis estadísticas: la hipótesis nula es que la tendencia central de las puntuaciones de las CE es la misma en los 6 estratos sociales $[\mathrm{H} 0$ : $\mathrm{E}(1)=\mathrm{E}(2)=\mathrm{E}(3)=\mathrm{E}(4)=\mathrm{E}(5)$ $=\mathrm{E}(6)]$; la hipótesis alterna es que la tendencia central de las puntuaciones es diferente entre algunos de los grupos $[\mathrm{H} 1: \mathrm{E}(1) \neq \mathrm{E}(2) \neq \mathrm{E}(3) \neq \mathrm{E}(4) \neq \mathrm{E}(5) \neq \mathrm{E}(6)]$.

El test de Kruskal-Wallis mostró una diferencia estadísticamente significativa según el estrato social, tanto en las puntuaciones totales del test $(H=17.335, g l=5, p=.004)$ como en las escalas SC $(H=23.909, g l=5, p<.000)$, HI $(H=20.789, g l=5, p=.001)$ y CC $(H=78.280, g l=5, p$ $<.000)$. En la escala VA no hay diferencias significativas $(H=2.950, g l=5, p=.708)$.

El análisis post-hoc indica que dichas diferencias ocurren en la puntuación total del test entre los estratos 1-3 $(U=$ $8331, Z=-3.342, p=.001, r=.133), 1-4(U=6653.5, Z=$ $-3.458, p=.001, r=.174), 1-5(U=2529.5, Z=-3.015, p$ $=.003, r=.223)$ y $1-6(U=1026.5, Z=-2.905, p=.004$, $r=.277)$. Los alumnos del estrato 1 exhiben creencias menos sofisticadas en todos los casos. En la escala SC hay diferencias entre los estratos 1-3 $(U=10383, Z=-3.058$, $p=.002, r=.134), 1-4(U=7288.5, Z=-3.937, p<.000$; $r=.193), 1-5(U=2992.5, Z=-3.219, p=.001, r=.228)$, $1-6(U=1069, Z=-3.127, p=.002 ; r=.293)$ y $2-4(U=$ 25736, $Z=-2.970, p=.003, r=.129)$. Nuevamente, los alumnos de estratos bajos ( 1 y 2 ) tienen creencias menos desarrolladas.

En la escala HI existen diferencias entre los estratos 3-4 $(U=59474.5, Z=-3.047, p=.002, r=.112)$, y $3-6(U=$ 9336.5, $Z=-3.469, p=.001, r=.158)$. El sentido de esta diferencia indica que los alumnos de estrato 3 tienen unas creencias más sofisticadas respecto a la habilidad innata que los de estratos 4 y 6 . En la escala $\mathrm{CC}$ hay diferencias entre los estratos 1-3 $(U=105109, Z=-3.344, p=.001$, $r=.145), 1-4(U=6343, Z=-5.442, p<.000, r=.263)$, $1-5(U=2426.5, Z=-4.811, p<.000, r=.340), 1-6(U=$ $919, Z=-4.519, p<.000, r=.414) ; \mathrm{y}$ también entre los estratos 2-3 $(U=33089.5, Z=-3.352, p=.001, r=.133)$,
2-4 $(U=20615.5, Z=-6.447, p<.000, r=.278), 2-5(U$

$=7751.5, Z=-5.266, p<.000, r=.316), 2-6(U=2875, Z$ $=-4.780, p<.000, r=.316), \mathrm{y}$ entre los estratos 3-4 $(U=$ $70767.5, Z=-4.427, p<.000, r=.153), 3-5(U=26683.5$, $Z=-3.218, p=.001, r=.131)$ y $3-6(U=9914, Z=-3.350$, $p=.001, r=.146$ ).

En general, se observan diferencias entre los estratos bajos $(1,2$ y 3$)$ y los altos $(4,5$ y 6$)$, estas diferencias indican unas creencias más elaboradas en los estratos más altos, excepto en el caso de la escala HI, en la que ocurre lo contrario.

Diferencias en las CE según el área de estudio

Hipótesis estadísticas: la hipótesis nula es que la tendencia central de las puntuaciones de las CE es la misma en las 6 áreas de estudio: 1. Ingeniería 2. Psicología 3. Filosofía 4. Filología 5. Economía 6. Bachillerato, $\left[\mathrm{H}_{0}: \mathrm{E}(1)=\mathrm{E}(2)\right.$ $=\mathrm{E}(3)=\mathrm{E}(4)=\mathrm{E}(5)=\mathrm{E}(6)]$; la hipótesis alterna es que la tendencia central es diferente entre algunos de los grupos $\left[\mathrm{H}_{1}: \mathrm{E}(1) \neq \mathrm{E}(2) \neq \mathrm{E}(3) \neq \mathrm{E}(4) \neq \mathrm{E}(5) \neq \mathrm{E}(6)\right]$.

La prueba de Kruskal-Wallis indica diferencias estadísticamente significativas según el área, tanto en las puntuaciones totales del test $(H=26.471, g l=5, p<.000)$, como en las escalas SC $(H=22.295, g l=5, p<.000)$, VA $(H=13.925, g l=5, p=.016)$ y CC $(H=126.458, g l=5$, $p<.000)$. En la escala $\mathrm{HI}$ no hay diferencias significativas $(H=5.457, g l=5, p=.363)$.

El análisis post-hoc indica que dichas diferencias ocurren en la puntuación total entre ingeniería-bachillerato $(U$ $=91533.5, Z=-4.631, p<.000, r=.150)$. En VA entre ingeniería-psicología $(U=49272, Z=-2.727, p=.007, r=$ $.098)$ e ingeniería- bachillerato $(U=117004.5 ; Z=-2.906$, $p=.004, r=.091)$. En la escala SC las diferencias ocurren entre las áreas de economía-bachillerato $(U=117004.5$, $Z=-2.906, p=.004, r=.091)$ y en la escala CC ocurren entre ingeniería-bachillerato $(U=8599 ; Z=-10.21, p<$ $.000 ; r=.316)$, psicología-bachillerato $(U=33096.5, Z$ $=-7.290, p<.000, r=.277)$, filosofía - bachillerato $(U=$ $391.5, Z=-3.488, p<.000, r=.158)$, filología-bachillerato $(U=2685.5, Z=-3.349, p=.001, r=.150)$ y economía- 
bachillerato $(U=7327, Z=-2.953, p=.003, r=.129)$. Las diferencias indican que los alumnos de bachillerato tienen creencias menos desarrolladas.

\section{DISCUSIÓN}

En el presente trabajo se realizó un estudio de las variables relacionadas con las CE en una muestra de estudiantes colombianos con el objetivo de profundizar en el conocimiento de factores que pueden influir en un desarrollo de las CE para generar programas de intervención educativa que propendan por la utilización de mejores estrategias de aprendizaje. En particular, se analizaron las diferencias en el desarrollo de las $\mathrm{CE}$ entre grupos de alumnos conformados por características socio-demográficas y educativas. Con respecto a las hipótesis planteadas al comienzo del estudio los resultados muestran el incumplimiento de la primera hipótesis, ya que los alumnos varones obtuvieron una mayor sofisticación en CC. Esto se opone a los resultados de Bendixen, Schraw y Dunkle (1998) y a los de Hofer (2000). Por otra parte, no hay diferencias significativas de acuerdo al sexo en otras sub-escalas, lo que apoya el patrón de resultados mixto señalado por Walter (2008).

En cuanto al tipo de colegio de procedencia públicoprivado, tampoco se ha podido mantener la hipótesis inicial. Siguiendo a Ismail et al. (2013) se esperaba que los alumnos de instituciones públicas tuvieran unas CE más desarrolladas. Según los resultados de este estudio ha ocurrido lo contrario: los alumnos de instituciones privadas presentan mayor desarrollo de las CE. Este resultado puede deberse a particularidades culturales, pues los colegios privados colombianos tienen más recursos económicos. Adicionalmente, los estudiantes de colegios privados suelen tener una mayor puntuación en el test de diagnóstico general del sistema educativo que cada año lleva a cabo el Instituto Colombiano para la Evaluación de la Educación (ICFES) en este país (Núñez, Steiner, Cadena y Pardo, 2002).

Este resultado pone en evidencia que tanto las posibilidades socio-económicas de los alumnos como un mejor desempeño en las pruebas ICFES son factores asociados con unas CE más desarrolladas. Lo anterior coincide con estudios previos en los que se ha encontrado que alumnos con mayores notas en tests generales de evaluación educativa exhiben una mayor sofisticación en sus CE (Hofer, 2000; Paulsen y Wells, 1998).

La hipótesis de que los estudiantes que habían repetido curso iban a tener unas creencias menos elaboradas tampoco se ha confirmado. Esto se puede deber a que los repetidores representan sólo el $20 \%$ de los participantes. Además, es importante tener en cuenta que ha podido transcurrir un tiempo desde el momento en que repitieron el curso; por lo tanto, es aconsejable medir esta variable solo en los alumnos que repitieron el curso recientemente.

Con respecto al sector de procedencia, se ha encontrado que no existen diferencias en las $\mathrm{CE}$ entre la procedencia rural o urbana. Esto es congruente con la revisión de la literatura, y más concretamente con el estudio de Topçu y Yilmaz (2009) en donde no había diferencias entre los estudiantes según el carácter rural o urbano del colegio al que asistían. Si bien los datos apoyan la hipótesis, sólo un $9 \%$ de la muestra provenía de un entorno rural.

Los resultados permiten mantener la hipótesis de que habría diferencias entre los alumnos según el nivel de estudios, teniendo los alumnos universitarios creencias más desarrolladas que los bachilleres, tendencia ha sido encontrada previamente en otras investigaciones (Baldwin y Alsumait, 2013; Walker, 2007).

Respecto a la influencia del nivel educativo de los padres sobre el desarrollo epistemológico de sus hijos, los resultados son congruentes con las hipótesis, ya que los alumnos cuyos progenitores tienen un nivel de primaria y bachillerato poseen unas creencias menos elaboradas en oposición los hijos de padres con un mayor nivel académico (universidad y posgrado). Esto confirma la importancia del rol familiar en el cambio epistemológico (Schommer 1990).

Siguiendo a Conley et al. (2004) y Ozkal et al. (2011), se supuso que habría una menor sofisticación en las CE de los estudiantes cuanto más bajo fuera el estrato socioeconómico al que pertenecieran. Los datos del presente estudio apoyan esta idea, aunque con matizaciones. Los estudiantes del estrato 1 tienen creencias más simples tanto en $\mathrm{CC}$ como en SC. En contraste, los estudiantes del estrato 6 tienen unas creencias menos sofisticadas en HI. Esto indica que los estudiantes de un estrato alto, al disponer de los recursos económicos suficientes, tienden a valorar menos el esfuerzo y a ver el aprendizaje como genéticamente aprendido (Chen y Pajares, 2010).

Por otra parte, es importante mencionar entre las limitaciones del presente estudio, que aunque se han encontrado diferencias significativas en algunas variables, estas tienen un tamaño del efecto moderado o bajo, lo cual permite afirmar que la magnitud estimada de las diferencias encontradas es débil. Esto supone un reto para la investigación en las $\mathrm{CE}$, ya que en ninguno de los trabajos previos consultados se han reportado los tamaños del efecto en las diferencias estimadas. Por lo tanto, cabe preguntarse si las diferencias encontradas en estudios previos son también débiles como en este caso.

Otra posible limitación es que la muestra, a pesar de ser grande, no ha sido seleccionada de forma probabilística, 
lo que limita la generalización de los resultados. Por ello, algunos desarrollos futuros se pueden centrar en el diseño de estudios con muestras probabilísticas y de carácter longitudinal para observar la trayectoria del desarrollo y del cambio epistemológico. Así mismo, se sugiere también estudiar otras poblaciones, tales como alumnos de postgrado o profesores. Se aconseja administrar el test a estudiantes que repitieron curso de forma más inmediata e incluir en la muestra alumnos que vayan a universidades públicas. Adicionalmente, puede ser interesante el desarrollo de más estudios que analicen la influencia de factores en las CE en diferentes países y culturas.

\section{REFERENCIAS}

Acosta, M. S. (2009). Los procesos de aprendizaje y su incidencia en la deserción estudiantil en el programa de química farmacéutica de la Universidad de Cartagena. Psicología desde el Caribe, 24, 26-58.

Alsamie, M. F. A. e Ismail, A. M. (2005). An investigation of the relationships between EFL pre-service teachers' epistemological beliefs and their learning strategies, teaching practices and foreign language classroom anxiety. Journal of Scientific Research in Education and Psychology, 18(3), 1-33.

Angeli, C. y Valanides, N. (2012). Epistemological beliefs and ill-structured problem-solving in solo and paired contexts. Journal of Educational Technology and Society, 15(1), 2-14.

Ato, M. López, J. J. y Benavente, A. (2013). Un sistema de clasificación de los diseños de investigación en Psicología. Anales de Psicología, 29(3), 1038-1059. doi: http://dx.doi. org/10.6018/analesps.29.3.178511

Aulls, M. W. y Lemay, D. (2013). Análisis de los beneficios y los resultados de aprendizaje de la Monografía del IB en la preparación de alumnos para estudios universitarios. $\mathrm{Ca}$ nadá: McGill University.

Baldwin, L. y Alsumait, D. (2013). The Impacts of Academic Levels and Information Science on Epistemological Beliefs of Undergraduate Students. En R. McBride y M. Searson (Eds.), Proceedings of Society Information Technology and Teacher Education International Conference 2013 (pp. 4635-4642). Chesapeake, VA.

Bendixen, L. D., Schraw, G. y Dunkle, M. E. (1998). Epistemic beliefs and moral reasoning. The Journal of Psychology, 132, 187-200.

Brownlee, J. M. (2003). Paradigm shifts in preservice teacher education students: A case study of changes in epistemological beliefs for teacher education students. Australian Journal of Educational and Developmental Psychology, 3, 1-6.

Buehl, M. M., Alexander, P. A. y Murphy, P. K. (2002). Beliefs about schooled knowledge: Domain specific or domain general? Contemporary Educational Psychology, 27, 415449. doi: $10.1006 /$ ceps.2001.1103
Cano, F. (2005). Epistemological beliefs and approaches to learning: Their change through secondary school and their influence on academic performance. British Journal of Educational Psychology, 75(2), 203-221.

Cano, F. y Cardelle, M. (2008). Family environment, epistemological beliefs, learning strategies, and academic performance: A path analysis. En M.S. Khine (Ed.), Knowing, knowledge, and beliefs: Epistemological studies across diverse cultures (pp. 219-240). New York: Springer.

Chan, K. W. y Elliott, R. G. (2002). Exploratory study of Hong Kong teacher education students' epistemological beliefs: Cultural perspectives and implications on beliefs research. Contemporary Educational Psychology, 27, 392-414. doi: 10.1006/ceps.2001.1102

Conley, A. M., Pintrich, P. R., Vekiri, I. y Harrison, D. (2004). Changes in epistemological beliefs in elementary science students. Contemporary Educational Psychology, 29(2), 186-204. doi: 10.1016/j.cedpsych.2004.01.004

Escalante, E. (2010). Propiedades psicométricas de un instrumento para medir creencias epistemológicas. Fundamentos en Humanidades, 21, 67-80.

Fritz, C. O., Morris, P. E. y Ritchler, J. J. (2012). Effect Size Estimates: Current Use, Calculations, and Interpretation. Journal Experimental Psychology, 141(1), 2-18.

García, M. y Sebastián, C. (2011). Creencias epistemológicas de estudiantes de pedagogía en educación parvularia, básica y media: ¿Diferencias en la formación inicial docente? Psykhe, 20(1), 29-43. doi: 10.4067/S071822282011000100003

Gómez, L. F. y Silas, J. C (2012). Las creencias epistemológicas de alumnos y profesores de $1^{\circ}$ de secundaria. Diálogos sobre educación, 3(5), 1-14.

Hernández, F. y Maquilón, J. J. (2011). Las creencias y las concepciones. Perspectivas complementarias. Revista electrónica interuniversitaria de formación del profesorado, $36(14), 165-175$.

Herrón, M. A. (2010). Epistemology and epistemic cognition: The problematic virtue of relativism and its implications for science education. Zona Próxima, 12, 96-107.

Hofer, B. K. (2000). Dimensionality and disciplinary differences in personal epistemology. Contemporary Educational Psychology, 25(4), 378-405. doi 10.1006/ceps.1999.1026

Hofer, B. K. y Pintrich, P. R. (1997). The development of epistemological theories: Beliefs about knowledge and knowing and their relation to learning. Review of Educational Research, 67(1), 88-140. doi: 10.3102/00346543067001088

Ismail, H., Hassan, A., Muhamad, M., Ali, W. Z. W., y Konting, M. (2013). Epistemological belief and learning approaches of students in higher institutions of learning in Malaysia. International Journal of Instruction, 6(1), 139-150.

Jehng, J. C., Johnsons, S. y Anderson, S. (1993). Schooling and students' epistemological beliefs about learning. Contem- 
porary Educational Psychology, 18(1), 23-25. doi 10.1006/ ceps.1993.1004

King, B. A. y Magun, S. (2009). Epistemological beliefs of engineering students. The Journal of Technology Studies, 35(2), 56-64.

Lahtinen, A. M. y Pehkonen, L. (2013). Seeing things in a new light: Conditions for changes in the epistemological beliefs of university students. Journal of Further and Higher Education, 37(3), 397-415. doi: 10.1080/0309877X.2011.645458

Llinares, I., García, A., Martí, M. y Casino, A. M. (2013). ¿Conocemos a nuestros estudiantes? Las creencias epistemológicas y el sistema de valores en el EEES. @ Tic. Revista D'innovació Educativa, 10, 24-32.

Martínez, R. D., Montero, Y. H. y Pedrosa, M. E. (2010). Creencias epistemológicas, estrategias de búsqueda de información y criterios para validar la información de la web. Revista Electrónica de Investigación Educativa, 12(1), 1-26.

Marton, F. (1981). Phenomenography: Describing conceptions of the word around us. Instructional Science, 10, 177-200.

Marzooghi R., Fouladchang, M. y Shemshiri, B. (2007). Gender and grade level differences in epistemological beliefs of Iranian undergraduate students. Journal of Applied Sciences, 24(7), 4698-4701. doi: 10.3923/jas.2008.4698.4701

Mason, L., Boldrin, A. y Zurlo, G. (2006). Epistemological understanding in different judgment domains: Relationships with gender, grade level, and curriculum. International Journal of Educational Research, 45, 43-56.

Núñez, J., Steiner, R., Cadena, X., y Pardo, R. (2002). ¿Cuáles colegios ofrecen mejor educación en Colombia? Archivos de Economía, 193, 1-56.

Önen, A. S. (2011). Investigation of students' epistemological beliefs and attitudes towards studying. Hacettepe Üniversitesi Eğitim Fakültesi Dergisi, 40, 300-309.

Ordóñez, X. G., Ponsoda, V., Abad, F. J. y Romero, S. J. (2009). Measurement of epistemological beliefs: Psychometric properties of the EQEBI test scores. Educational and Psychological Measurement, 69, 287-302. doi:10.1177/0013164408323226

Ozkal, K., Tekkaya, C., Sungur, S., Cakiroglu, J., y Cakiroglu, E. (2011). Elementary students' scientific epistemological beliefs in relation to socio-economic status and gender. Journal of science teacher education, 22(2), 115-127.

Özkan, S. y Tekkaya, C. (2011). How do epistemological beliefs differ by gender and socio-economic status? HU Journal of Education, 41, 339-348.

Paechter, M., Rebmann, K., Schloemer, T., Mokwinski, B., Hanekamp, Y. y Arendasy, M. (2013). Development of the Oldenburg Epistemic Beliefs Questionnaire (OLEQ), a German questionnaire based on the Epistemic Belief Inventory (EBI). Current Issues in Education, 16(1), 1-17.

Paulsen, M. B. y Wells, C. T. (1998). Domain differences in the epistemological beliefs of college students. Research in Higher Education. 39(4), 365-384.
Pozo, J. I. y Scheuer, N. (1999). Las concepciones sobre el aprendizaje como teorías implícitas. En: J. I. Pozo y C. Monereo (Coord.), El aprendizaje estratégico. Cap. 4, 87-108. Madrid: Santillana.

Rodríguez, L. (2005). Análisis de las creencias epistemológicas, concepciones y enfoques de aprendizaje de los futuros profesores (Tesis Doctoral no publicada). Universidad de Granada, Granada.

Säljö, R. (1979). Learning in the learners' perspective. I: Some common sense conceptions. Reports from the Institute of Education, University of Göteborg, 76.

Schommer, M. (1990). Effects of beliefs about the nature of knowledge on comprehension. Journal of Educational Psychology, 82(2), 498-504. doi: 10.1037/00220663.82.3.498

Schommer, M., Beuchat, M. y Hernández, F. (2012). Creencias epistemológicas y de aprendizaje en la formación inicial de profesores. Anales de Psicología, 28(2), 465-474. doi: 10.6018/analesps.28.2.125341

Schommer, M., Calvert, C., Gariglietti, G. y Bajaj, A. (1997). The development of epistemological beliefs among secondary students: A longitudinal study. Journal of Educational Psychology, 89(1), 37-40.

Schommer, M. y Dunnell, P. A. (1994). A comparison of epistemological beliefs between gifted and non-gifted high school students. Roeper Review, 16(3), 207-210. doi:10.1080/02783199409553575

Schommer, M. y Orpha, K. (2013). Domain specific and general epistemological beliefs: Their effects on mathematics. Revista Investigación Educativa, 31(2), 317-330. doi: 10.6018/rie.31.2.170911

Taha, M. y El-Habbal, M. (2013). The relationship between epistemic beliefs and academic performance: Are better students always more mature? Journal of Educational and Developmental Psychology, 3(1), 158-172. doi: 10.5539/ jedp.v3n1p158

Terrazas, W. S. y Frenay, M. (2009). Creencias epistemológicas: ¿Cómo comprender su relación con la epistemología? Papeles salmantinos de educación, 13, 95-120.

Terzi, A. R., Çetin, G. y Eser, H. (2012). The relationship between undergraduate students' locus of control and epistemological beliefs. Educational Research, 3(1), 30-39.

Topçu, M. S. y Yilmaz, T. Ö. (2009). Elementary students' metacognition and epistemological beliefs considering science achievement, gender and socioeconomic Status. Ilkogretim Online, 8(3), 676-693. doi: 10.1007/s10972-009-9169-0

Vizcaíno, A., Otero, I y Mendoza, Z. (2013). Las creencias epistemológicas como alternativa para la comprensión del aprendizaje. Psicoespacios, 7(11), 117-163.

Walker, D. L. (2007). The Development and Construct Validation of the Epistemological Beliefs Survey for Mathematics (Tesis doctoral). Oklahoma State University. , Oklahoma, U.S.A. 